Part 3. The Interstellar Medium 


\title{
PHYSICAL PROCESSES IN THE LARGE SCALE ISM FROM DUST OBSERVATIONS
}

\author{
F. BOULANGER \\ Institut d'Astrophysique Spatiale, Université Paris, France
}

\begin{abstract}
Over the last two decades observations of dust emission in the infrared have played an important role in the development of research on the interstellar medium. The study of the spectral energy distribution has led to the discovery of small dust particles including the large aromatic molecules (PAHs). Infrared sky images have been used to study the structure of interstellar matter, the evolution of dust within the interstellar medium and the star formation efficiency of interstellar clouds.
\end{abstract}

\section{Introduction}

In this conference we have been given a panorama of sky-surveys available over the whole electromagnetic spectrum. Most of these have contributed to our understanding of the interstellar medium (ISM) but biased by my own research experience, I review physical processes in the ISM from the perspective opened by the analysis of dust emission in infrared sky images. Since the interpretation of these observations has made use of data at other wavelengths, this topic illustrates well the benefits of data intercomparison across the electromagnetic spectrum. I will mostly refer to observations obtained with the Infrared Astronomy Satellite (IRAS), the Cosmic Background Explorer (COBE) and the Infrared Space Observatory (ISO). But data obtained with other instruments or space projects like the japanese satellite IRTS and the Air-Force project MSX (Price, this volume, p. 115) are also relevant. A general description of surveys in the infrared is given by Beichman in this volume (p. 27).

In the $100 \mu \mathrm{m}$ IRAS images, diffuse emission with a filamentary structure reminiscent of cirrus clouds is present over most of the sky. In this review I 
will show that cirrus observations have provided new insights about many key processes in the evolution of interstellar matter. In particular, the composition of dust (Section 2) and its evolution within the interstellar medium, the small-scale structure of clouds and the chemical transition between atomic and molecular gas (Section 3). A key motivation of research on interstellar matter is to understand physical parameters controlling the onset and the efficiency of star formation. Infrared Surveys in the mid and far infrared provide an important statistical information on star formation which I also discuss in the paper (Section 4).

\section{Dust Composition}

\subsection{EMISSION SPECTRUM}

Infrared observations of ordinary interstellar matter, located far away from any peculiar heating source, have been mostly obtained from space with cooled telescopes because the emission is faint and extended. These observations, in particular those of cirrus clouds optically thin to Galactic star light, constitute a reference for the study of dust emission properties. In Figure 1, I have gathered data providing the complete spectral energy distribution of dust in the Solar Neighborhood heated by the local mean interstellar radiation field. The emission power which is distributed over a large range of wavelengths from the near-infrared to millimeter wavelengths, originates from particles emitting over a wide range of temperatures. To account for the spread in temperatures it is necessary to extend the size distribution of dust grains down to molecules with a few tens of atoms (Désert et al. 1990, Dwek et al. 1997).

At long wavelengths the emission spectrum from large grains is well fit by a single Planck curve with an emissivity proportional to $\nu^{2}$ and $\mathrm{T}_{\mathrm{d}}=17.5 \mathrm{~K}$ (Boulanger et al. 1996a). The dust emissivity,

$\tau_{\lambda} / \mathrm{N}_{\mathrm{H}}=1.010^{-25}(\lambda / 250 \mu \mathrm{m})^{-2} \mathrm{~cm}^{2}$ for $\lambda>250 \mu \mathrm{m}$, is remarkably close to the value obtained by Draine and Lee (1984) for a mixture of compact graphite and silicate grains and is much smaller than values predicted for porous or fractal grains (Wright 1987, Mathis and Whiffen 1989). At wavelengths shorter than $100 \mu \mathrm{m}$ the observed emission is well in excess of the single temperature fit. This excess emission is attributed to particles small enough to be heated to temperatures much higher than the equilibrium temperature of the large grains by the stochastic absorption of photons. The presence of such small particles was first advocated on the basis of near and mid-IR observations of the Galactic plane and reflection nebulae (Sellgren et al. 1983). By providing complete sky maps of the near and mid-IR emission from interstellar matter, IRAS and DIRBE have demonstrated the ubiquity and importance of these small particles. Léger and 
Puget (1984) associated the emission from small particles with the presence of a well defined set of emission bands at $3.3,6.2,7.7,8.6$ and $11.3 \mu \mathrm{m}$ in the spectra of a wide range of celestial objects. These emission bands being characteristic of $\mathrm{C}-\mathrm{C}$ and $\mathrm{C}-\mathrm{H}$ bonds in aromatic molecules, they proposed that the smallest particles are large polycyclic aromatic hydrocarbons (PAHs). It is now experimentally demonstrated that the $12 \mu \mathrm{m}$ diffuse emission measured by IRAS is associated with PAHs. The presence of the 3.3 and $6.2 \mu \mathrm{m}$ emission features in the spectrum of the diffuse emission from the Galaxy was first evidenced by the Arome balloon experiment (Giard et al. 1994, Ristorcelli et al. 1994). Spectra from the Galactic plane emission obtained recently with the ISO and IRTS satellites show the presence of the full set of PAHs features (Mattila et al. 1996, Onaka et al. 1996, Tanaka et al. 1996). The instruments on board of ISO are also sufficiently sensitive to detect the emission bands in the emission from high latitude cirrus clouds (Boulanger et al. 1996b and 1997). The presence of all the known emission features from aromatic hydrocarbon in the spectrum of the Galaxy and nearby clouds strongly supports the existence of large aromatic molecules in the most general physical conditions in interstellar clouds. The abundance of Carbon in PAHS with less than a few 100 atoms is estimated to represent $15 \%$ of the total cosmic abundance of carbon.

The temperature fluctuations associated with the quantization of the heating radiation significantly affects the emission spectrum of grains up to sizes of $50 \AA$ (Draine and Andersson 1985). Particles in the intermediate size range between PAHs and large dust grains are often referred to as very small grains (VSGs). For the local interstellar radiation field, VSGs emission dominates the emission from other dust components from 15 to $60 \mu \mathrm{m}$. Since no emission feature has yet been observed in this wavelength range the chemical nature of VSGs is unknown. However, intermediate size grains must be related to the PAHs and the large grain. It is thus speculated that the VSGs could be small coal like particles (carbon clusters with variable degrees of hydrogenation and mixed hybridation, Papoular et al. 1996) which can be seen as aggregates of PAH molecules. Another possibility is that the size distribution of silicate grains extends down to small sizes. Infrared spectroscopy is a powerful mean to identify dust composition. The analysis of the spectroscopic information obtained with ISO should thus lead to significant advances in our knowledge of the chemical composition of dust.

\subsection{EVOLUTION OF DUST WITHIN THE ISM}

Data on scattering, absorption and emission from dust grains provide independent evidence for the evolution of interstellar dust from place to place in 


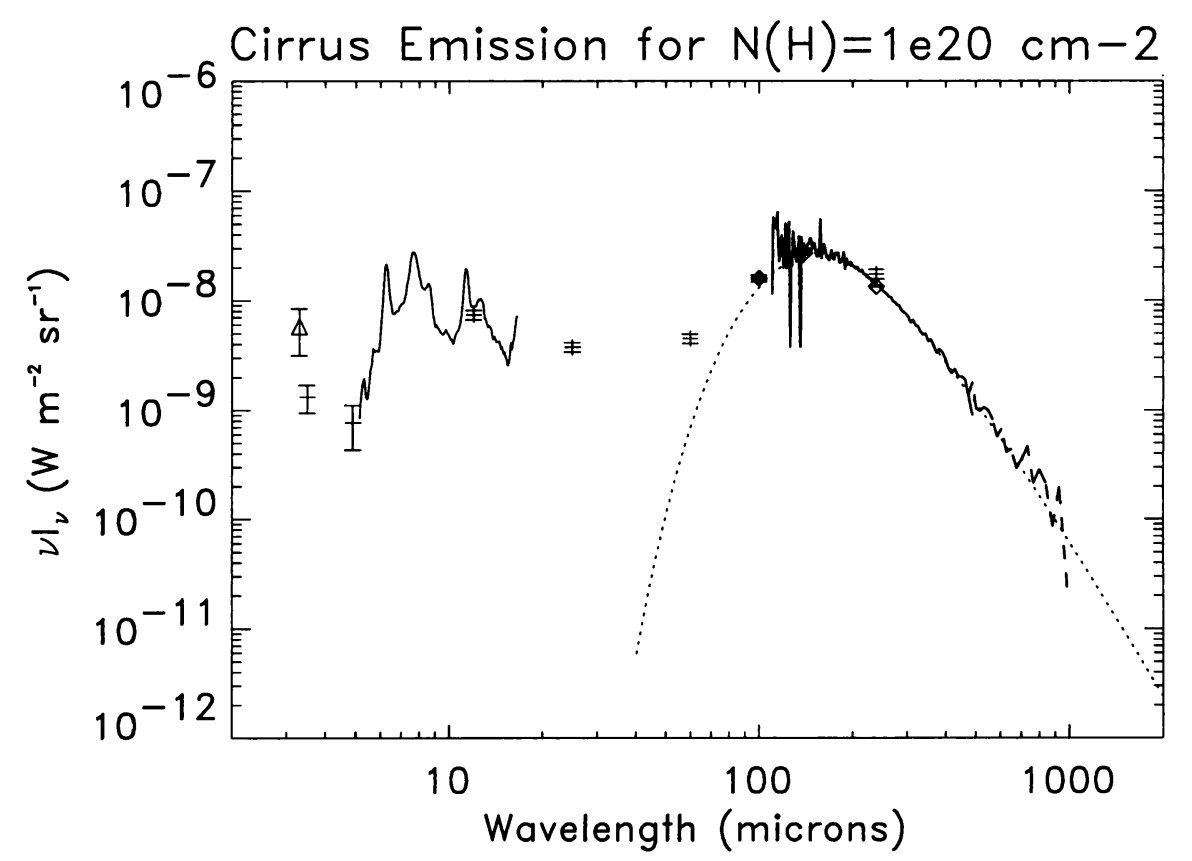

Figure 1. The dust emission spectrum from the near-IR to millimeter wavelengths. This figure combines (1) the $3.3 \mu \mathrm{m}$ emission of cirrus associated with the molecular ring (Giard et al. 1994), (2) an ISOCAM spectrum between 5 and $16 \mu \mathrm{m}$ measured at the northern edge of the dense cloud in Ophiuchus (Boulanger et al. 1996b), (3) measurements of the high latitude cirrus emission from the COBE satellite with the Diffuse Infrared Background Experiment (DIRBE) (Bernard et al. 1996) and the Far Infrared Absolute Spectrometer (FIRAS) (Boulanger et al. 1996a).

the interstellar medium. The interstellar extinction curve is known to exhibit considerable variations in the UV and optical from one line of sight to another. An important conclusion of the analysis of the IRAS and COBE all-sky images is that there is substantial evidence for changes in the dust size distribution and emission properties within clouds of moderate overall opacity ( $\mathrm{A}_{\mathrm{V}}<2 \mathrm{mag}$ ) to stellar light (Weiland et al. 1986, Boulanger et al. 1990, Laureijs et al. 1991). The emission from PAHs and very small grains in the 12,25 and $60 \mu \mathrm{m}$ bands of IRAS, relative to that from large dust grains at $100 \mu \mathrm{m}$, varies by one order of magnitude from cloud to cloud and within clouds. Models of the IR emission from clouds show that these color variations trace changes in the abundance of PAHs and very small particles (Bernard et al. 1993). The spectral studies made with the IRAS data have been extended to the whole dust emission spectrum with the COBE data. Lagache et al. (1997) have shown that, where the abundance of small dust particles is low, the equilibrium temperature of large grains is 
lower than the mean cirrus value. This is an interesting result showing that the change in abundance of very small grains is correlated with a change in the temperature of large grains. With an angular resolution of a few arcminutes observations made with the balloon-borne instrument Pronaos have led to the detection of dense condensations in nearby clouds where the dust temperatures is as low as $12 \mathrm{~K}$ (Ristorcelli et al. 1997).

The infrared color variations have been correlated with changes in the UV part of the extinction curve (Boulanger et al. 1994). However, the amplitude of none of the spectral features in the extinction curve is linearly proportional to the abundance of the mid-IR emitters. This means that none of these spectral features is specific to the mid-IR emitters which is not unexpected since the size range of particles susceptible to contribute to spectral features in the UV extinction, up to sizes of a few $100 \AA$, is much larger than that of mid-IR emitters (size $<30 \AA$ ).

Two processes are assumed to play an important role in the evolution of dust within the interstellar medium. First, model calculations suggest that dust in the low density components of the ISM is efficiently destroyed by supernovae shock waves. The efficiency of this process is demonstrated by the large variations of depletions between the different phases of the ISM (e.g., Sofia and Cardelli 1994). Recent theoretical work (Jones et al. 1996) and observations (Zagury et al. 1997) suggest that the shattering of grains in grain-grain collisions is an effective process to produce small particles. The effect of this process on the grain size distribution has only been investigated in fast shocks $(100 \mathrm{~km} / \mathrm{s}$ and higher $)$ traveling in the low density intercloud phase of the ISM Jones et al. estimate the velocity threshold for grain shattering in grain-grain collisions to be as low as $1 \mathrm{~km} / \mathrm{s}$. Since this velocity is typical of the relative motions between dust particles induced by turbulent motions (Falgarone and Puget 1995) grain shattering could also be an effective process to form small particles within interstellar clouds. Second, investigations based on dust absorption features seen in the infrared indicate that dust grains accrete gas molecules in dense clouds and that icy mantles are able to evolve chemically towards refractory compounds through photolysis. It is most likely that this evolution process also affects the small particles which can grow in size by mutual coagulation or can stick on large grains. Serra et al. (1992) and Marty et al. (1994) have suggested that in dense clouds PAH molecules could be bound together by metals and thus be in the form of organometallic complexes. This is a possible explanation of the observed correlation between the cold component of the infrared emission (the component with a low abundance of small particles) with the ${ }^{13} \mathrm{CO}$ emission from dense molecular gas (Laureijs et al. 1991, Abergel et al. 1994). 


\section{Infrared Cirrus}

\subsection{INFRARED EMISSION TRACER OF INTERSTELLAR GAS}

Among the various observational means of imaging local interstellar matter: optical images (Guhathakurta and Cutri 1994), $21 \mathrm{~cm}$ emission from H I (see Kulkarni and Heiles 1987), and CO surveys at high latitude (Magnani et al. 1996), the IRAS images remain unique in providing a complete view at the intricate structure of this matter over a wide range of angular scales and brightness. In this section we describe the observational basis which supports the use of the far-IR brightness as a tracer of both atomic and molecular gas within cirrus.

Several studies based on the IRAS data showed that the $100 \mu \mathrm{m}$ emission away from the Galactic plane and away from the principal nearby molecular complexes is generally tightly correlated with the distribution of the neutral atomic gas (e.g., Boulanger and Pérault 1988; Deul and Burton 1993). This correlation analysis has been extended to longer wavelengths using the DIRBE and FIRAS data (Boulanger et al. 1996). The far-infrared spectrum and data points in Figure 1 are the result of this correlation in regions of $\mathrm{HI}$ column density smaller than $510^{20} \mathrm{~cm}^{-2}$. In these low column density regions, the $\mathrm{I}_{\nu}(100 \mu \mathrm{m})$ emission for $\mathrm{N}(\mathrm{HI})=10^{20} \mathrm{H} \mathrm{cm}^{-2}$ is $0.85 \mathrm{MJy} / \mathrm{sr}$ and $0.54 \mathrm{MJy} / \mathrm{sr}$ for the IRAS and DIRBE data, respectively. The two numbers differ due to a known calibration difference between the two experiments. The far IR-HI correlation shows that dust and gas are well mixed and that, at high latitude, the intensity of dust heating is quite uniform. The emission ratio also provides an empirical conversion factor between far-infrared brightness and gas column density.

The validity of the far-IR emission as a tracer of gas distribution has been extended to regions of higher column density by comparing the 100 $\mu \mathrm{m}$ brightness with the visible extinction. Most published studies apply to clouds at intermediate latitudes with moderate extinctions, Av up to 2-3 mag (e.g., Boulanger et al. 1997). The $\mathrm{I}_{\nu}(100 \mu \mathrm{m}) / \mathrm{A}_{\mathrm{v}}$ values found in these translucent molecular clouds are 2 to 3 times lower than the mean value of $15.9 \mathrm{MJy} / \mathrm{sr}$ per mag for atomic clouds at high latitude (IRAS value). The reduction factor fits with estimates of the effect of the radiation field attenuation on the IR emission from non-transparent clouds (Bernard et al. 1993).

\subsection{ATOMIC AND MOLECULAR GAS IN CIRRUS}

Gas in Cirrus is dominantly atomic but CO observations (e.g., Magnani et al. 1996) and the comparison between IRAS and H I data suggest that many cirrus features contain some molecular gas. The presence of molecular 
gas is often inferred from the existence of an infrared excess emission with respect to what is expected from the atomic gas seen in emission in the $\mathrm{H} \mathrm{I}$ $21 \mathrm{~cm}$ line (Désert et al. 1988, Boulanger et al. 1996). In the all-sky studies with 30 to $40^{\prime}$ resolution many high latitude molecular clouds are heavily diluted. The comparison between IRAS, $\mathrm{HI}$ and $\mathrm{CO}$ observations is thus the most conclusive where $\mathrm{H} \mathrm{I}$ observations with resolution comparable or better than IRAS have been obtained (e.g., Herter et al. 1990, Joncas et al. 1992). With higher angular resolution one may clearly identify clouds for which the IR-H I correlation extends down to the IRAS resolution and others for which there is no correlation (e.g., de Vries et al. 1987). Many of the latter cirrus coincide with high latitude molecular clouds seen in emission in CO (e.g., Heithausen et al. 1993).

The $\mathrm{CO}$ emission is far from being simply related to the column density of molecular hydrogen in cirrus. Based on a large and deep CO survey of cirrus clouds around the North Celestial Pole, Heithausen et al. (1993) showed that there is not a one to one correlation between the two intensities and that there is no threshold of $\mathrm{I}_{\nu}(100 \mu \mathrm{m})$ for the detection of CO. Several studies have concluded to the existence of a gas component seen in the infrared but not in emission in H I nor in CO (Reach et al. 1994, Meyerdierks and Heithausen 1996). For example, the ratio between gas mass as derived from the far-infrared emission and the $\mathrm{CO}$ emission varies by one order of magnitude among clouds in the Chameleon complex (Boulanger et al. 1997). We want to stress that the reported variations between molecular mass and $\mathrm{CO}$ emission are local in nature and that they do not question large-scale estimates of molecular masses derived from $\mathrm{CO}$ data. In particular, the mass of molecular gas in the Solar Neighborhood derived from $\mathrm{CO}$ observations is known to be in good agreement with that inferred from Copernicus measurements of $\mathrm{H}_{2}$ absorption lines in the UV (Savage et al. 1977).

Measurements of the $\mathrm{CO}$ column density, together with investigations of physical conditions indicate that cirrus clouds span the critical regime where the abundance of $\mathrm{CO}$ starts to become significant but in which most of the carbon is still in atomic form (van Dishoeck et al. 1991, Gredel et al. 1992). Theoretical models (van Dishoeck and Black 1988 and Viala et al. 1988) show that in this regime, small fluctuations in involved physical parameters can lead to large variations in observable CO line intensity. The abundance of $\mathrm{CO}$ depends not only on the total column density but also on the gas density, the far-UV radiation field and the dust extinction in the far-UV. Various observations suggest that the presence of dense gas is correlated with an excess in the far-UV extinction curve (Cardelli and Clayton 1991, Jenniskens et al. 1992, Boulanger et al. 1994). Dust may thus facilitate the onset of formation of self-shielded $\mathrm{CO}$ by attenuating 
radiation coinciding with photo-dissociative transitions. It is most likely that this is only one of the many processes which connect the dynamical, thermal and chemical evolutions of interstellar matter.

\subsection{DENSITY STRUCTURE}

Most cirrus studies concentrate on localized features and little is known about the large scale organization of cirrus. The lack of systematic distance estimates has prevented so far the construction of a 3-D representation of the nearby interstellar medium which will locate cirrus clouds with respect to the local cavities of coronal gas and the energy sources, stellar winds and supernovae explosions, which have created them. This knowledge may be important to understand the morphology of cirrus clouds and the origin of their turbulent motions. The X-ray all-sky maps from ROSAT have been used to identify several instances of shadows due to the absorption of Xray emission from the coronal gas by intervening cirrus clouds (Mebold et al. 1994). The Hipparcos catalog (Mignard, this volume, p. 399) contains 30,000 stars with measured parallax in between 100 and $200 \mathrm{pc}$ for the Sun. This distance information combined with the stellar reddenings opens the prospect of better understanding the large scale organization of cirrus. The three dimension structure of cirrus could also be mapped by combining the Hipparcos data with IRAS searches for heated dust around stars (Gaustad and van Buren 1993).

The infrared sky images from both IRAS and DIRBE have been used to characterize statistically the self-similar structure of interstellar matter. Gautier et al. (1992) have used IRAS images to measure the power spectrum of high latitude cirrus clouds. The shapes of the power spectra were found to be well represented by a power law. Two dimensional analyses yield indices near -3 from several tens of degrees to the $5^{\prime}$ angular resolution of IRAS (Gautier et al. 1992, Kogut et al. 1996). Based on these results, Gautier et al. deduced that the brightness contrast (i.e, the column density contrast for the matter traced by the far-infrared emission) increases with angular size, $\theta$, as $\theta^{0.5}$. But Gautier et al. also realized that the most striking features of the IRAS images correspond to a non-Gaussian tail in the distribution of brightness fluctuations. Thus, the sky structure is not fully characterized by power spectra which only measure the standard deviation of the brightness fluctuations. Abergel et al. (1996) went further in this analysis by using wavelet transforms which allow to study the scaling law between brightness fluctuations and angular size as a function of position in the image. This work shows that the power law breaks at the position of filaments which have an excess of power on small angular sizes. In the image analyzed by Abergel et al., these singular structures correspond to 
regions of cold IRAS emission which are known from the correlation with ${ }^{13} \mathrm{CO}$ emission to trace dense gas. Observations of molecular lines at radio wavelengths provide a complementary perspective on the structure of cirrus clouds. The $\mathrm{CO}$ emitting gas has been shown to be structured on scales much smaller than the IRAS resolution. Small scale clumping is also necessary to explain the observed excitation of the $\mathrm{CO}$ rotational transitions. Gas densities in $\mathrm{CO}$ emitting pieces of cirrus are estimated to be at least $10^{3} \mathrm{H}_{2} \mathrm{~cm}^{-3}$ (Falgarone et al. 1991 and van Dishoeck et al. 1991). The excitation of other molecules such as CS provides even higher density estimates in some cirrus cores (Reach et al. 1995). The minimal density value of $10^{3} \mathrm{H}_{2} \mathrm{~cm}^{-3}$ is one to two orders of magnitude larger than the mean densities one gets from the mean column densities and structure sizes derived from the IRAS images, especially where $\mathrm{CO}$ is detected and the mean column density of gas as estimated from $\mathrm{I}_{\nu}(100 \mu \mathrm{m})$ is low. The velocity information provided by radio observation has also been used to show that the turbulent energy within cirrus clouds greatly exceeds the binding energy of gravity (e.g., Heithausen 1966). The existence of a self similar structure over many decades of sizes with large density contrast down to unresolved scales is a fascinating aspect of cirrus clouds which remains to explained.

\section{Star Formation}

In the Galactic Plane the infrared emission is made of a diffuse component and localized sources respectively accounting for $3 / 4$ and $1 / 4$ of the IR luminosity of the Galaxy.

The various groups which have analyzed the IRAS data (Pérault 1987, Cox and Mezger 1988, Sodroski et al. 1989, Bloemen et al. 1990) and more recently the DIRBE data (Sodroski et al. 1997) agree on the following conclusions. The heating of Galactic dust is dominated by $\mathrm{O}, \mathrm{B}$ and $\mathrm{A}$ stars. Older stars contribute only $30 \%$ of the overall luminosity of the Galaxy. A large fraction of the luminosity of dust is provided by stars of intermediate mass, late B and A stars with ages in the range $10^{7}$ to a few $10^{8} \mathrm{yrs}$. This is an important conclusion which implies that the IR emission provides an information on star formation distinct from that of radio observations measuring the thermal emission from $\mathrm{H}$ II regions. From the Solar Neighborhood to the molecular ring, the radial distribution of the summed luminosity of $\mathrm{O}, \mathrm{B}$ and $\mathrm{A}$ derived from the infrared data follows that of ionizing stars derived from radio observations. The enhanced rate of star formation currently observed in the molecular ring has thus been lasting for at least a few $10^{7}$ years: it is not a recent burst.

The brightest sources were known from balloon observations prior to the IRAS survey (Hauser et al. 1984 and references therein). They are 
all associated with known luminous star forming regions (e.g., Myers et al. 1987). IRAS allowed the discovery of a large number of ultra-compact H II regions which represent the youngest high mass star in the Galaxy (Wood and Churchwell 1989). These stars have been found to concentrate in an annulus of massive star formation coincident but about $30 \%$ narrower in radius than the Molecular Ring (Bronfman et al. 1996). But, since only a minor fraction of molecular clouds in the Galaxy is associated with high mass stars, little is known about star formation efficiency for the bulk of the molecular gas in the Galaxy. The ISO satellite has opened the possibility to extend this statistical study to lower mass stars.

In the wavelength range $5-17 \mu \mathrm{m}$ ISOCAM, the camera of the Infrared Space Observatory (ISO) is able to detect sources more than 100 times fainter than IRAS. One of the major research goals being pursued with this new instrument is the study of low mass star formation. With the available sensitivity it is possible to detect pre-main sequence stars of Solar mass within the Molecular ring, the region of highest concentration of gas and star formation, in the Galaxy. The preliminary analysis of ISOCAM images of the Galactic plane (Pérault et al. 1996) shows that half of the detected sources have no near-infrared counterpart in the $\mathrm{K}$ images of the DENIS survey, the near-IR southern sky survey being carried out at La Silla. The only plausible candidates for such a large number of cold sources $\left(1300 / \mathrm{deg}^{2}\right)$ are dusty young stars. It is foreseen that ISO observations in the Galactic plane will allow to detect 50,000 new young stars and thus provide statistical information about star formation efficiency and luminosity function of young stars for a large set of molecular ring clouds. Another interesting result of the ISOCAM observations is the detection of dark features which are likely to be due to absorption of the diffuse Galactic emission by opaque gas condensations. The most contrasted features would correspond to very dense filaments with visible extinction Av of at least 25 mag. This provides another interesting perspective on star formation since many of this absorbing structures could contain proto-stellar condensations.

\section{Conclusion}

Infrared surveys have opened new perspectives on interstellar matter. The emission from dust has been observed and mapped from the near-IR to millimeter wavelengths. The spectral energy distribution and spectroscopy have been used to investigate the size distribution and composition of interstellar dust and its evolution within the interstellar medium. The IRAS sky images have revealed the intricate structure of interstellar matter with a yet unsurpassed horizon in terms of sky coverage and sensitivity. These 
maps have prompted numerous studies of the structure of interstellar clouds at other wavelengths. In particular, $\mathrm{CO}, \mathrm{H} \mathrm{I}$ and optical observations have broadened the IRAS perspective by providing images on smaller scales and velocity information. Soon these observations will be compared with images obtained with ISO. The infrared surveys also provide a census of young embedded stars allowing to statistically study the star formation efficiency and the stellar luminosity function for a large set of clouds.

Since a significant fraction of the conference was devoted to the analysis of large data-bases, I want to stress that the problems raised by the analysis and intercomparison of extended emission from Galactic interstellar matter are different in nature from those raised by the study of catalogs. Research is also badly needed to develop tools to statistically characterize and compare images.

\section{References}

Abergel, A., Boulanger, F., Mizuno, A. and Fukui, Y. 1994, Astrophys.J., 423, L59

Abergel, A., Boulanger, F., Delouis, J.M., Dudziak, G., Steindling, S. 1996, Astron.Astrophys., 309, 245

Bernard, J.P. et al. 1996, in Unveiling the Cosmic Infrared Background, ed. E. Dwek, AIP Conf. Proceedings 348, p. 105

Bernard, J.P., Boulanger, F., and Puget, J. L. 1993, Astron.Astrophys., in press

Bloemen, J.B.G.M., Deul, E. R., and Thaddeus, P. 1990, Astron.Astrophys., 233, 437

Boulanger, F. and Pérault , M. 1988, Astrophys.J., 330, 964

Boulanger, F., Falgarone, E., Puget, J.L., and Helou, G. 1990, Astrophys.J., 364, 136

Boulanger, F., Prévot, M. L., and Gry, C. 1994, Astron.Astrophys., 285, 956

Boulanger, F. et al. 1996a, Astron.Astrophys.312, 256

Boulanger, F. et al. 1996b, Astron.Astrophys.315, L325

Boulanger, F., Bronfman, L., Dame, T.M. and Thaddeus, P. 1997, Astron.Astrophys., in press

Bronfman, L., Nyman, L.A., amd May, J. 1996, Astron.Astrophys.Suppl., 115, 1

Cardelli, J. A. and Clayton, G. C. 1991, Astron.J., 101, 1021

Cox, P. and Mezger, P. G. 1989, Astron.Astrophys.Rev. 1, 49

Désert , F. X., Bazell, D. and Boulanger, F. 1988, Astrophys.J., 334, 815

Désert , F. X., Bazell, D. and Blitz, L. 1990, Astrophys.J., 355, L51

Deul, E. R. and Burton, W. B. 1990, Astron.Astrophys., 230, 153

de Vries, H. W., Heithausen, A. and Thaddeus, P. 1987, Astrophys.J., 319, 723

Draine, B. T., and Lee, H. M. 1984, Astrophys.J., 285, 89

Draine, B.T. and Anderson, N. 1985, Astrophys.J.292, 494

Dwek, E. et al. 1997, Astrophys.J.475, 565

Falgarone, E., Phillips, T. G. and Walker, C. K. 1991, Astrophys.J., 378, 186

Falgarone, E., Puget, J.L. 1995, Astron.Astrophys.293, 840

Gaustad, J. E., van Buren, D. 1993, Publ.Astron.Soc.Pacific105, 1127

Gautier, T.N., Boulanger, F., Pérault, M., Puget, J.L. 1992, Astron.J., 103, 1313

Giard, M.,Lamarre, J.M., Pajot, F., Serra, G. 1994, Astron.Astrophys., 286, 203

Gredel, R., van Dishoeck, E. F., de Vries, C. P. and Black, J. H. 1992, Astron.Astrophys., 257,245

Hauser, M.G. et al. 1984, Astrophys.J.285, 74

Heithausen, A., Stacy, J. G., de Vries, H. W., Mebold, U. and Thaddeus, P. 1993, Astron.Astrophys., 268, 265. 
Heithausen, A. 1996, Astron.Astrophys.314, 251

Herter, T., Shupe, D. L. and Chernoff, D. F. 1990, Astrophys.J., 352, 149

Jenniskens, P., Ehrenfreund, P. and Désert, F. X. 1992, Astron.Astrophys., 265, L1

Joncas, G., Boulanger, F., Dewdney, P. E. 1992, Astrophys.J., 397, 165

Jones, A.P., Tielens, A.G.G.M. and Hollenbach, D.J. 1996, Astrophys.J.469, 740

Kogut, A. et al. 1996, Astrophys.J.460, 1

Lagache, G., Abergel, A., Boulanger, F., Puget, J.L. 1997, Astron.Astrophys.in press

Laureijs, R. J., Clark, F. O., and Prusti, T. 1991, Astrophys.J., 372, 185

Léger, A., Puget, J.L. 1984, Astron.Astrophys.137, L5

Magnani, L. Hartmann, D., Speck, B.G. 1996, Astrophys.J. Supp.106, 447

Marty, P., Serra, G., Chaudret, B. and Ristorcelli, I. 1994, Astron.Astrophys.282, 916

Mathis, J.S. and Whiffen, G. 1989, Astrophys.J.341, 808

Mattila, K. et al. 1996, Astron.Astrophys.315, L353

Mebold, U., Kerp, J., Moritz, P. Engelmann, J. and Herbstmeier, U. 1994 in The First

Symposium on the Infrared Cirrus and Diffuse Interstellar Clouds, R.M. Cutri and W.B. Latter (eds), ASP Conf. Ser. 58, 45.

Meyerdierks, H., Heithausen, A. 1996, Astron.Astrophys.313, 929

Myers, P.C., et al. 1986, Astrophys.J.301, 398

Onaka, T., Yamamura, I., Tanabe, T., Roellig, T., Yuen, L. 1996, Publ.Astron.Soc.Japan 48, L41

Papoular, R., et al. 1996, Astron.Astrophys.315, 222

Pérault , M. 1987, Thèse d'Etat, Université Paris VII

Pérault , M. et al. 1996, Astron.Astrophys.315, L165

Puget, J.L. and Léger, A. 1989, Ann.Rev.Astron.Astrophys. 27, 161

Reach, W. T., Koo, B. C., Heiles, C. 1994, Astrophys.J., 429, 672

Reach, W.T., Pound, M.W., Wilner, D.J., and Lee, Y. 1995, Astrophys.J.441, 244

Ristorcelli, I. et al. 1994, Astron.Astrophys.286, L23

Ristorcelli, I. et al. 1997, Diffuse Infrared Radiation and the IRTS, ed. T. Matsumoto

Savage, B.D., Bohlin, R.C., Drake, J.F., Budich, W. 1977, Astrophys.J.216, 291

Sellgren, K., Werner, M. W. and Dinerstein, H. L. 1983, Astrophys.J., 271, L13

Serra, G. et al. 1992, Astron.Astrophys.260, 489

Sofia, U.J., Cardelli, J.A. and Savage, B.D. 1994, Astrophys.J.430, 650

Sodroski, T.J. et al. 1997, Astrophys.J.480, 173

Tanaka, M., Matusmoto, T., Murakami, H., Kawada, M., Noda, M. Matsuura, S. 1996, Publ.Astron.Soc.Japan 48, L53

van Dishoeck, E. F., and Black, J. H. 1988, Astrophys.J., 334, 771

van Dishoeck, E. F., Black, J. H., Phillips, T. G., and Gredel, R. 1991, Astrophys.J., 366,141

Viala, Y. P., Letzelter, C., Eidelsberg, M. and Rostas, F. 1988, Astron.Astrophys., 193, 265

Weiland, J.L., Blitz, L., Dwek, E., Hauser, M. G., Magnani, L., and Rickard, L. J. 1986,Astrophys.J., 306, L101

Wood D.O.S., Churchwell, E. 1989 Astrophys.J.340, 265

Wright, E.L. 1987, Astrophys.J.320, 818

Zagury, F., Boulanger, F., and Jones, A.P. 1997, Astron.Astrophys.in press 